\title{
Nobiletin Protects Endothelial Cell Function via Upregulation of eNOS/ET-1 and Antioxidant Status-Related Genes under Nonstimulated and Inflammatory Conditions
}

\author{
Lu Li $\mathbb{D},{ }^{1,2}$ Biyun Zhang $\mathbb{D}^{2},{ }^{2}$ Ludovica De Pieri, ${ }^{2}$ Yue Wu, ${ }^{2}$ Karen Birch $\mathbb{D},{ }^{3}$ \\ and Christine Boesch $\mathbb{D D}^{2}$ \\ ${ }^{1}$ Beijing Advanced Innovation Center for Food Nutrition and Human Health, School of Food and Health, \\ Beijing Technology and Business University, Beijing 100048, China \\ ${ }^{2}$ School of Food Science and Nutrition, University of Leeds, Leeds LS2 9JT, UK \\ ${ }^{3}$ School of Biomedical Sciences, University of Leeds, Leeds LS2 9JT, UK \\ Correspondence should be addressed to Lu Li; lil@btbu.edu.cn and Christine Boesch; c.bosch@leeds.ac.uk
}

Received 15 September 2021; Revised 12 January 2022; Accepted 20 January 2022; Published 8 February 2022

Academic Editor: Chunpeng Wan

Copyright $\odot 2022 \mathrm{Lu} \mathrm{Li}$ et al. This is an open access article distributed under the Creative Commons Attribution License, which permits unrestricted use, distribution, and reproduction in any medium, provided the original work is properly cited.

Nobiletin, a natural polymethoxylated flavonoid compound, has been shown to exert a wide range of biological activities. However, there is limited evidence on the molecular mechanisms by which nobiletin modulates endothelial cell function. Our aim was to investigate the potential of nobiletin to enhance endothelial cell functionality under nonstimulated and inflammatory conditions. To this end, gene expression relevant to nitric oxide (NO) production, endothelin 1 (ET-1), and antioxidant status in human endothelial cells EA.hy926 was determined using real-time PCR. The fluorescence probe 4,5-diaminofluorescein (DAF-2) was used to measure NO production. The results demonstrated significant upregulation of eNOS and antioxidant genes as well as downregulation of ET-1 by nobiletin under nonstimulated and TNF- $\alpha$-stimulated inflammatory conditions. These findings suggest a promising protective effect of nobiletin with relevance for cardiovascular health, likely through the NO/ET-1 dynamics and Nrf2 signaling pathway.

\section{Introduction}

Epidemiological studies have suggested that the intake of citrus fruits is inversely associated with the risk of ischemic stroke [1] and coronary heart disease [2]. Citrus fruits, such as oranges, grapefruit, lemons, limes, mandarins, bergamots and pomelos, are abundant in flavonoids, including hesperidin, hesperetin, naringin, naringenin, diosmin, quercetin, rutin, nobiletin, and tangeretin [3]. Polymethoxyflavones (PMFs) such as nobiletin and tangeretin represent the characteristic compounds of the genus [4]. Nobiletin has attracted wide research attention for its various beneficial properties, such as antioxidant [5], anti-inflammatory [6], and anticancer [7] effects. According to epidemiological evidence, the consumption of citrus flavonoids is inversely associated with the risk of cardiovascular mortality [8].
Given the crucial role of endothelial function in the development of cardiovascular disease [9], the effect of citrus flavonoids on endothelial function is of great importance. However, findings of randomized controlled trials (RCTs) investigating how orange juice consumption modulates endothelial function are inconsistent [10]. A recent crossover RCT demonstrated that two-week consumption of blood orange juice improved flow-mediated dilation in healthy overweight and obese participants [11]. Flavanonerich citrus beverages alleviated the transient decline in postprandial endothelial function in healthy men following a sequential double meal rich in fat [12]. However, orange juice did not acutely affect endothelial function in men at moderate risk of cardiovascular disease [13]. Therefore, the mechanisms by which citrus flavonoids modulate endothelial function remain to be elucidated. 
Endothelial dysfunction is defined as a reduction in nitric oxide (NO) production and/or bioavailability, with or without an imbalance between relaxing and contracting factors associated with elevated inflammation [14]. As the major vasodilator produced by endothelial cells, NO plays a pivotal role in maintaining vascular homeostasis. Hence, endothelial nitric oxide synthase (eNOS) function and activity are crucial for endothelial cell functionality [15]. The potent contracting factor endothelin 1 (ET-1) produced by endothelial cells has been reported to reduce eNOS promoter activity and eNOS protein levels, thereby contributing to decreased levels of NO [16]. The imbalance between NO and ET-1 contributes to endothelial dysfunction [17]. Administration of resveratrol reversed a high-cholesterol dietinduced downregulation of $\mathrm{NO}$ and upregulation of ET-1 in experimental hypercholesterolemic rabbits [18]. There is, however, a paucity of data regarding how citrus flavonoids regulate ET-1 expression. Of note, excessive oxidative stress contributes to elevated eNOS uncoupling and reduced NO bioavailability [19]. Therefore, redox balance in endothelial cells is of great importance. The transcription factor nuclear factor erythroid 2-related factor 2 (Nrf2) regulates the expression of antioxidant genes such as heme oxygenase-1 (HO-1), gamma-glutamyl cysteinyl synthetase (GCLC), and superoxide dismutase (SOD) which contribute to the maintenance of cellular redox balance [20]. Notably, endothelial cell dysfunction is a trigger or consequence of oxidative stress and inflammation [14].

Therefore, in this study, we investigated the effect of citrus flavonoids, with a focus on nobiletin, on eNOS, ET-1, and antioxidant gene expression under both nonstimulated and inflammatory conditions.

\section{Materials and Methods}

2.1. Reagents. Hesperetin, naringenin, and phorbol-12myristate-13-acetate (PMA) were obtained from SigmaAldrich (Dorset, UK). Nobiletin, tangeretin, and resveratrol were purchased from Extrasynthese (Genay, France). The purity of all compounds was above 97\%. Dulbecco's phosphate-buffered saline (DPBS) without calcium and magnesium and fetal bovine serum (FBS) were purchased from Thermo Fisher Scientific (Loughborough, UK). Dulbecco's modified Eagle's medium (DMEM), L-glutamine, penicillin-streptomycin, and trypsin-EDTA were obtained from Lonza (Slough, UK). TRIsure reagent and SensiMix ${ }^{\mathrm{TM}}$ were obtained from Bioline (London, UK). The iScript ${ }^{\mathrm{TM}}$ cDNA synthesis kit was purchased from Bio-Rad (Hertfordshire, UK). Chloroform, diethylpyrocarbonate (DEPC)treated (RNase-free) water, and propan-2-ol were obtained from Thermo Fisher Scientific. All other chemicals and reagents were obtained from Sigma-Aldrich unless specified otherwise.

2.2. Cell Culture and Treatments. The experiments were conducted using human endothelial cells EA.hy926 (CRL$2922^{\mathrm{TM}}$ ) which were obtained from the American Type Culture Collection (ATCC). Cells were cultured in DMEM with $1 \mathrm{~g} / \mathrm{L}$ of glucose, $2 \mathrm{mML}$-glutamine, $1 \mathrm{mM}$ sodium pyruvate, $10 \%$ fetal bovine serum, and $1 \%$ penicillin and streptomycin in a humidified atmosphere with $5 \% \mathrm{CO}_{2}$ at $37^{\circ} \mathrm{C}$. EA.hy 926 cells were seeded at a density of $1 \times 10^{5}$ cells/ $\mathrm{mL}$ and left to grow overnight to reach $90 \%$ confluence. Stock solutions of flavonoids were prepared in 100\% dimethyl sulfoxide (DMSO) at $100 \mathrm{mM}$ in aliquots, with the exception of tangeretin (at $25 \mathrm{mM}$ due to its limited solubility in DMSO). Dilutions were made from stock solutions to specific concentrations immediately prior to treatment. Initially, optimisation of NOS3 induction was conducted by testing effects of different incubation periods $(6 \mathrm{~h}$ and $18 \mathrm{~h})$, glucose content $(1 \mathrm{~g} / \mathrm{L}$ and $4.5 \mathrm{~g} / \mathrm{L})$, and FBS (0\% and $10 \%)$, with resveratrol $(25$ and $50 \mu \mathrm{M})$ and PMA $(10 \mathrm{nM})$ as positive controls [21]. Following NOS3 optimisation, cells were incubated with naringenin, hesperetin, nobiletin, and tangeretin at different concentrations for $18 \mathrm{~h}$ in FBS-free, low-glucose medium $(1 \mathrm{~g} / \mathrm{L})$.

2.3. Cell Viability. The potential cytotoxicity of test compounds was determined by the neutral red (NR) assay as described previously [22]. Specifically, varying concentrations $(0,25,50$, and $100 \mu \mathrm{M})$ of compounds in phenol redfree and serum-free medium were prepared in triplicate and incubated at $37^{\circ} \mathrm{C}$ for $18 \mathrm{~h}$. After medium removal, cells were incubated with medium containing NR at $40 \mu \mathrm{g} / \mathrm{mL}$ for $2 \mathrm{~h}$. Subsequently, cells were washed with DPBS. The NR dye was extracted using destain solution, and absorbance was read at $540 \mathrm{~nm}$ using a plate reader (Tecan SPARK 10M, Männedorf, Switzerland). The viability of the treated cells was calculated as percent relative to control.

2.4. RNA Extraction and Real-Time PCR. RNA was extracted from EA.hy926 cells using TRIsure and quantified using NanoDrop2000 (Thermo Fisher Scientific, with purity assessed by A260/280. Reverse transcription was conducted using the iScript ${ }^{\mathrm{TM}}$ cDNA synthesis kit. Real-time PCR was performed using SensiMix from Bioline ${ }^{\mathrm{TM}}$ on a StepOne Real-Time PCR system (Thermo Fisher Scientific. Primers were designed using NCBI tools (Table 1) as previously described [23], which were synthesized by Eurofins Genomics (Ebersberg, Germany).

2.5. Measurement of Nitric Oxide. The fluorescence probe 4,5-diaminofluorescein (DAF-2) was used to determine nitric oxide production by EA.hy926 cells following $18 \mathrm{~h}$ incubation with different test compounds. Measurements were conducted as previously reported [24]. Following the removal of the cell culture medium and a wash with DPBS, cells were incubated with L-arginine $(100 \mu \mathrm{M})$ in DPBS for $10 \mathrm{~min}$. Subsequently, cells were incubated with DAF-2 $(0.1 \mu \mathrm{M})$ in the dark for $5 \mathrm{~min}$. Fluorescence was measured at room temperature with excitation wavelength at $485 \mathrm{~nm}$ and emission wavelength at $520 \mathrm{~nm}$ with a Tecan plate reader. Autofluorescence of DAF-2 was subtracted from the total fluorescence. 
TAble 1: Primer sequences of target genes for real-time PCR.

\begin{tabular}{lcccc}
\hline Gene & Accession number & Annealing temp. $\left({ }^{\circ} \mathrm{C}\right)$ & Forward primer & Reverse primer \\
\hline ACTB & P60709 & 55 & AGAGCTACGAGCTGCCTGAC & AGCACTGTGTTGGCGTACAG \\
NOS3 & P29474 & 57.3 & GCAGCCTCACTCCTGTTTTC & GGTCTTCTTCCTGGTGATGC \\
HMOX1 & P09601 & 58 & CTTCTTCACCTTCCCCAACA & AGCTCCTGCAACTCCTCAAA \\
GCLC & P48506 & 58 & CAATGGGAAGGAAGGTGTGT & GCGATAAACTCCCTCATCCA \\
EDN1 & P05305 & 56 & GATGCCAATGTGCTAGCCAA & GCTGTTTCTCATGGTCTCCG \\
\hline
\end{tabular}

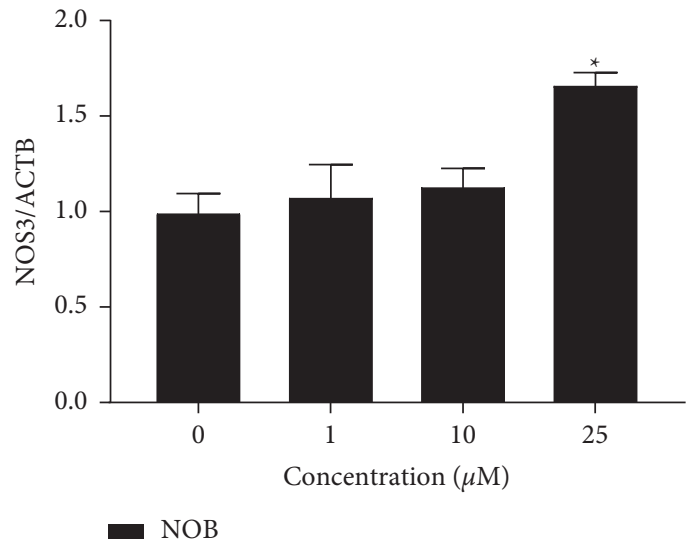

(a)

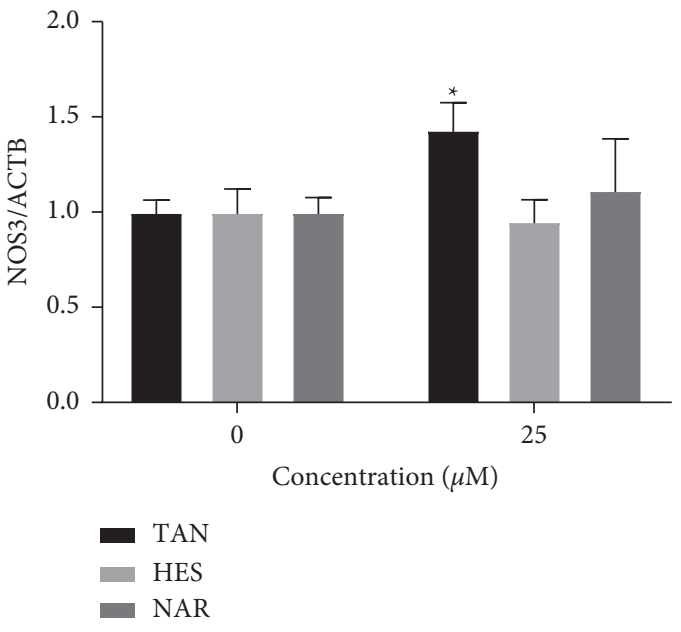

(b)

FIGURE 1: Effect of increasing concentrations of citrus flavonoids: (a) nobiletin (NOB) and (b) tangeretin (TAN), hesperetin (HES), and naringenin (NAR) on NOS3 mRNA levels. Cells were incubated for $18 \mathrm{~h}$ with concentrations up to $25 \mu \mathrm{M}$. Data are presented as mean \pm SD. *indicates significant differences between the treatment group and untreated control group at $p<0.05$.

2.6. Measurement of Reactive Oxygen Species. The generation of reactive oxygen species (ROS) was determined using the fluorescent probe dihydrodichlorofluorescein ( $\left.\mathrm{H}_{2} \mathrm{DCF}-\mathrm{DA}\right)$. Confluent endothelial cells in 96-well plates were incubated with $20 \mu \mathrm{M} \mathrm{H}_{2} \mathrm{DCF}-\mathrm{DA}$ for 30 min under standard conditions. Cells were then washed with DPBS and incubated with increasing concentrations of nobiletin $(0,1,5$, and $10 \mu \mathrm{M})$ for $30 \mathrm{~min}$ followed by the addition of $250 \mu \mathrm{M}$ hydrogen peroxide for $1 \mathrm{~h}$. Fluorescence intensity was measured at $485 \mathrm{~nm}$ and $535 \mathrm{~nm}$ excitation and emission wavelengths, respectively, using a Tecan plate reader in well-scanning mode. Results were calculated in percent of stimulated control cells.

2.7. Statistical Analysis. Results of three independent passages were expressed as mean \pm standard deviation (SD). Statistical analysis was conducted using Statistical Package for the Social Sciences (SPSS, version 24, IBM Corporation, USA). Treatment effects were analyzed by one-way analysis of variance (ANOVA) followed by the post hoc test of Dunnett's (comparing multiple treatments to a single control). $p<0.05$ was considered as statistically significant.

\section{Results and Discussion}

3.1. Differential Effects of Citrus Flavonoids on eNOS Expression. The concentrations used in the following experiments were not toxic to cells (Figure S1). Cell viability

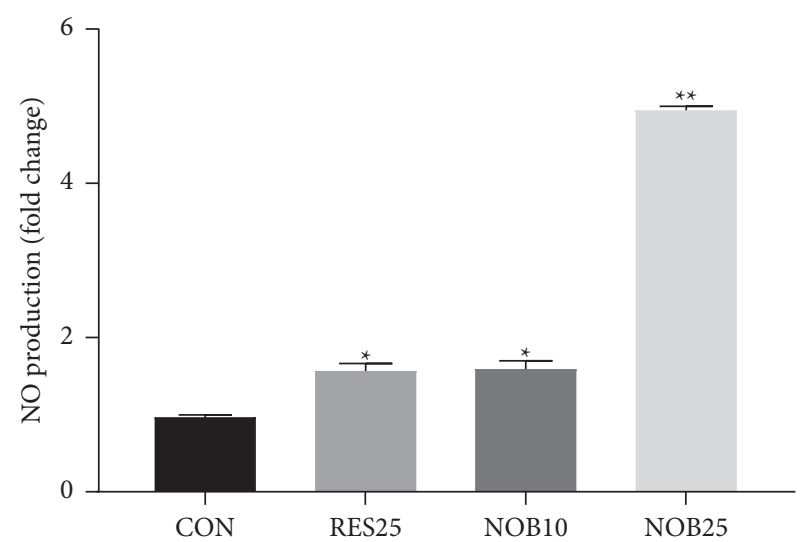

FIGURE 2: Dose-dependent effect of nobiletin to increase NO production using the DAF-2 assay. Effect of increasing DAF-2 detected NO release from EA.hy926 cells after $18 \mathrm{~h}$ incubation with nobiletin (NOB) at 10-25 $\mu \mathrm{M}$ and resveratrol (RES; $25 \mu \mathrm{M}$ ) as a positive control. Data were expressed as a fold change of control. Data are presented as mean $\pm \mathrm{SD} .{ }^{*}$ and ${ }^{* *}$ indicate significant differences between the treatment group and untreated control group at $p<0.05$ and $p<0.01$, respectively. CON: control.

did not differ between the DMSO-treated group $(98.8 \pm 3.1 \%)$ and untreated group $(100 \pm 2.1 \%)(p=0.71)$; therefore, DMSO used in experiments at $0.4 \%$ or lower, was not considered to have a negative effect on cell viability. In order to establish NOS3 induction, EA.hy926 cells were incubated with PMA, a known inducer of NOS3 mRNA and 
protein levels [25]. As shown in Figure S2, PMA exposure resulted in a dose-dependent induction from 0 to $1000 \mathrm{nM}$ which was significantly higher at $1-1000 \mathrm{nM}$ with maximum induction at $10 \mathrm{nM}$ compared to control. Induction of NOS3 mRNA following incubation with PMA or resveratrol was stronger at $18 \mathrm{~h}$ than $6 \mathrm{~h}$ (Figure S3). Hence, an incubation period of $18 \mathrm{~h}$ was selected to evaluate effect of citrus flavonoids on NOS3 mRNA expression.

In the next step, the potential of nobiletin, tangeretin, naringenin, and hesperetin to induce NOS3 mRNA was evaluated at concentrations ranging from 1 to $25 \mu \mathrm{M}$. As shown in Figure 1(a), nobiletin incubation led to a significant increase in NOS3 at $25 \mu \mathrm{M}$. Compared to resveratrol which had only resulted in a marked induction at $50 \mu \mathrm{M}$ (Figure S3), nobiletin was more potent to induce NOS3 mRNA expression at a lower concentration. NOS3 mRNA expression was also significantly upregulated by tangeretin at $25 \mu \mathrm{M}$ (Figure 1(b)). NOS3 mRNA expression was not significantly changed by hesperetin and naringenin (Figure 1(b)).

NO production was significantly augmented by nobiletin in a concentration-dependent manner, from 1.6-fold upregulation at $10 \mu \mathrm{M}$ to 5 -fold upregulation at $25 \mu \mathrm{M}$ (Figure 2). Resveratrol was used as a positive control for NO induction resulting in a 1.6 -fold NO production at $25 \mu \mathrm{M}$. Therefore, nobiletin appeared to be more potent in inducing $\mathrm{NO}$ than resveratrol when compared at $25 \mu \mathrm{M}$.

Since NO plays a pivotal role in endothelial function, the regulation of its synthesis by citrus flavonoids is crucial to the understanding of their effects on cardiovascular health. Importantly, eNOS expression can be regulated at different levels, such as eNOS promoter activity, eNOS transcription and mRNA stability, and posttranslational regulation [26]. The current study investigated effects on eNOS at the mRNA level and NO release.

Treatment with hesperetin $(12.5-100 \mu \mathrm{M})$ for $24 \mathrm{~h}$ dosedependently upregulated NO production in human umbilical vein endothelial cells (HUVECs), whereas treatment with naringenin did not result in significant changes in $\mathrm{NO}$ production under the same conditions [27]. Additionally, hesperetin $(50 \mu \mathrm{M}, 24 \mathrm{~h})$ significantly increased eNOS transcription and protein expression in HUVECs whereas no significant changes were observed following treatment with naringenin [27]. In contrast, incubation with hesperetin and naringenin for $18 \mathrm{~h}$ did not change eNOS mRNA levels in EA.hy926 cells in the present study, suggesting cell typeand/or time point-associated differences [28]. Whilst some citrus flavonoids might not modulate eNOS expression, they might contribute to the improvement of cardiovascular health by different mechanisms [29].

Polymethoxylated flavones have been highlighted for their anti-inflammation [30] and anticancer effects [31] in recent years. However, the effects of nobiletin and tangeretin on eNOS expression in human endothelial cells have not been investigated. In comparison, nobiletin and tangeretin were found to be more potent to induce eNOS mRNA levels in our study compared to hesperetin and naringenin. This could be attributed to the presence of the double bond at the $2-3$ position in the $\mathrm{C}$ ring of nobiletin and tangeretin, which has been associated with an upregulation in eNOS mRNA expression [32], potentially due to the reduction potential of the double bond. Among the investigated citrus flavonoids in the present study, nobiletin was the most effective compound in increasing eNOS mRNA and NO production under the defined conditions. Consistent with these results, nobiletin significantly increased the relaxation of phenylephrine-induced contraction in endothelium-intact rat aorta [33]. In support of this observation, nobiletin has been shown to promote the phosphorylation of eNOS at position Ser-1177 by increasing endothelial $\left[\mathrm{Ca}^{2+}\right] \mathrm{i}$, thereby increasing NO production and vasodilation in phenylephrineprecontracted mesenteric arteries isolated from rats [34]. In keeping with these findings, nobiletin alleviated iron overload damage in vascular endothelium through increasing eNOS phosphorylation and NO production [35]. In addition, nobiletin was found to induce endothelium-independent vasodilation in rat aorta. Specifically, nobiletin inhibited phenylephrine-mediated contraction of endothelium-denuded rat aorta through the activation of $\mathrm{Ca}^{2+}-\mathrm{ac}_{-}$ tivated $\mathrm{K}^{+}(\mathrm{BK})$ channel and ATP-sensitive $\mathrm{K}^{+}\left(\mathrm{K}_{\mathrm{ATP}}\right)$ channel, leading to vasodilation via membrane hyperpolarization [33]. Taken together, nobiletin has been shown to exert vasodilator effects by different mechanisms, such as inducing eNOS expression, promoting eNOS phosphorylation as well as activating $\mathrm{BK}$ and $\mathrm{K}_{\mathrm{ATP}}$ channels. Although the phosphorylation level of eNOS at Ser-1177 upon treatment of nobiletin was not measured in this study, a recent study has found a striking discordance between eNOS phosphorylation and NO formation in endothelial cells [36]. The phosphorylation of eNOS as a surrogate marker for activation of the eNOS enzyme can therefore be questioned. The generation of $\mathrm{NO}$ was determined as the ultimate outcome to confirm the transcriptional increase in eNOS mRNA levels in the present study, although it cannot be ruled out that other mechanisms are contributing to NO increases.

\subsection{Differential Effects of Citrus Flavonoids on Antioxidant} Status-Related Genes. Modulation of HMOX1 and GCLC mRNA expression by citrus flavonoids was also evaluated. Nobiletin significantly augmented HMOX1 (Figure 3(a)) and GCLC (Figure 3(b)) mRNA expression at $25 \mu \mathrm{M}$, whereas HMOX1 and GCLC mRNA levels were not changed by tangeretin, hesperetin, and naringenin.

Elevated ROS levels lead to a reduction in eNOS cofactor $\mathrm{BH}_{4}$ and facilitate eNOS uncoupling, thereby reducing NO bioavailability [37]. Hence, antioxidant status plays a crucial role in maintaining endothelial cell function and NO/ET-1 balance. Several proteins/enzymes contribute to maintaining cellular redox balance, including HO-1 and GCLC; however, there is limited research that has considered induction of HO- 1 and GCLC by nobiletin and tangeretin in human endothelial cells. The nobiletin-dependent upregulation of HO-1 and GCLC as observed in the current study is suggesting improvement of antioxidant status, thereby supporting findings on the beneficial effects of nobiletin on eNOS and NO production. Present results are in agreement 


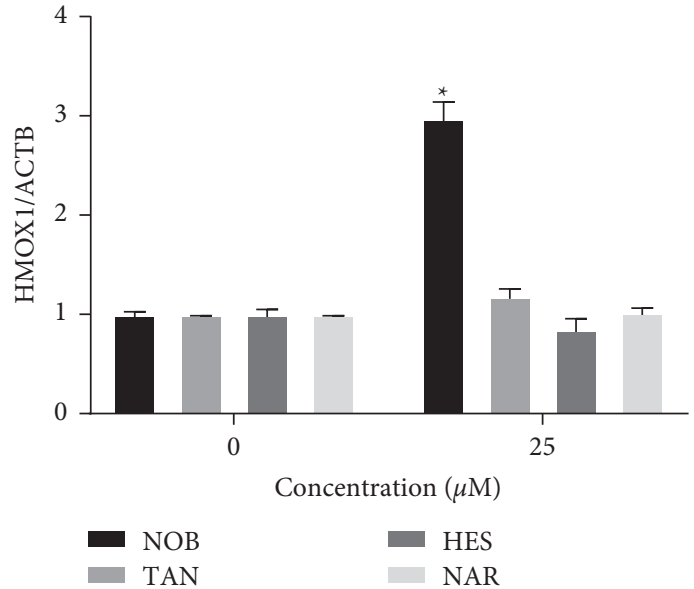

(a)

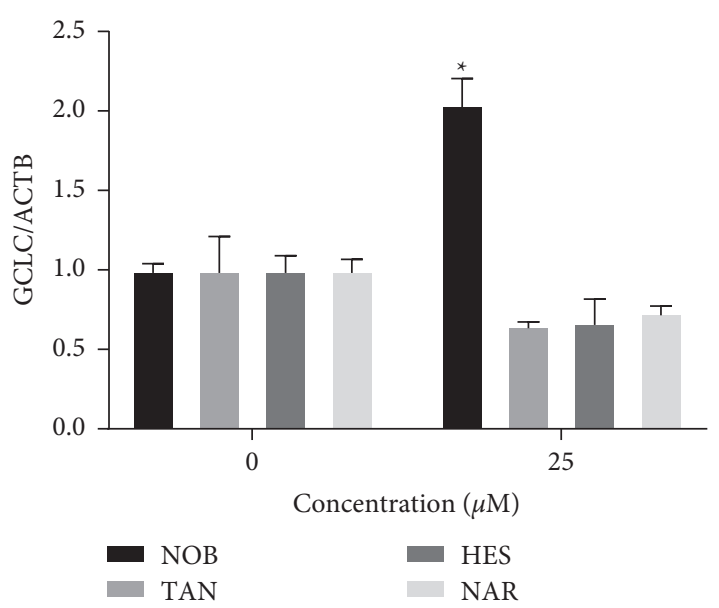

(b)

Figure 3: Effect of nobiletin (NOB), tangeretin (TAN), hesperetin (HES), and naringenin (NAR) on HMOX1 (a) and GCLC (b) mRNA levels after $18 \mathrm{~h}$ incubation. Data are presented as mean $\pm \mathrm{SD} .{ }^{*}$ indicates significant differences between the treatment group and untreated control group at $p<0.05$.

with a previous study that showed upregulation of Nrf-2/ HO-1 and MMP pathways following nobiletin administration and amelioration of vascular alterations in L-NAMEinduced hypertensive rats [38]. Furthermore, incubation with nobiletin $(0-10 \mu \mathrm{M})$ resulted in a dose-dependent reduction of hydrogen peroxide-stimulated ROS levels in EA.hy926 cells (Figure 4), confirming the contribution of nobiletin to enhancing and/or stabilizing cellular antioxidant status. The beneficial effect of nobiletin on ROS levels has also been demonstrated in vivo in mice fed with a highfat diet [39]. In addition, the chemical structure of citrus flavonoids indicates their role as radical scavengers and hydrogen-donating antioxidants [40]. Altogether, citrus flavonoids such as nobiletin can not only act as antioxidants to scavenge free radicals but can also upregulate antioxidant status-related gene expression to maintain cellular redox state and limit cell damage. Although the effect on antioxidant enzymes HO-1 and GCLC was not confirmed on protein level in this study, the reduced ROS levels are supportive to the improvement of antioxidant status in endothelial cells, likely through direct and indirect mechanisms.

\subsection{Dose-Dependent Inhibition of ET-1 mRNA Expression by} Nobiletin. Given that nobiletin was the most potent compound among the test compounds to induce NOS3, HMOX1, and GCLC mRNA expression, its impact on ET-1 mRNA expression was also investigated. ET-1 mRNA levels were reduced by nobiletin in a concentration-dependent manner, with a $35 \%$ decrease at $25 \mu \mathrm{M}(p<0.05)$ (Figure 5).

Hesperetin and naringenin at $1 \mu \mathrm{M}$ significantly reduced ET-1 mRNA expression in $\mathrm{H}_{2} \mathrm{O}_{2}$-treated HUVECs whereas no effect was observed on NOS3 mRNA expression [32]. The present study was the first to demonstrate that nobiletin $(1-25 \mu \mathrm{M})$ resulted in a concentration-dependent upregulation of NOS3 expression and downregulation of ET-1 in human endothelial cells, providing new insight into how

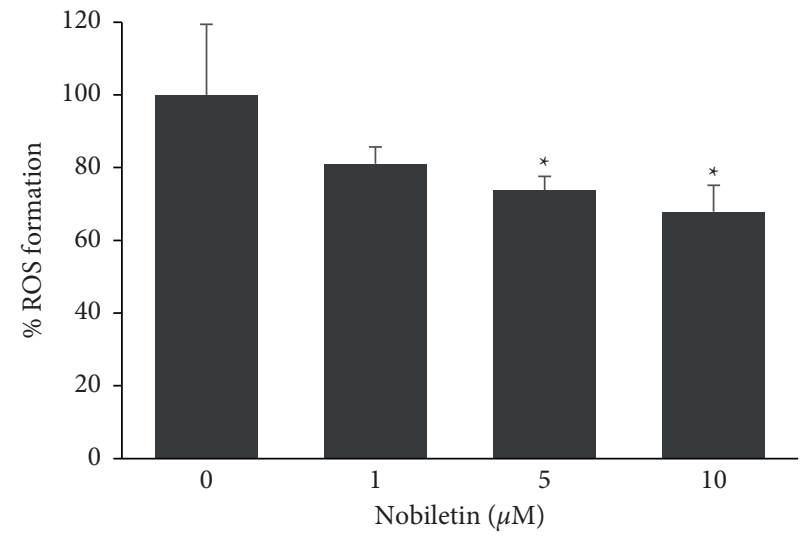

Figure 4: Effect of nobiletin $(0-10 \mu \mathrm{M})$ on hydrogen peroxideinduced ROS formation. Data are presented as mean \pm SD. *indicates significant differences between the treatment group and untreated control group at $p<0.05$.

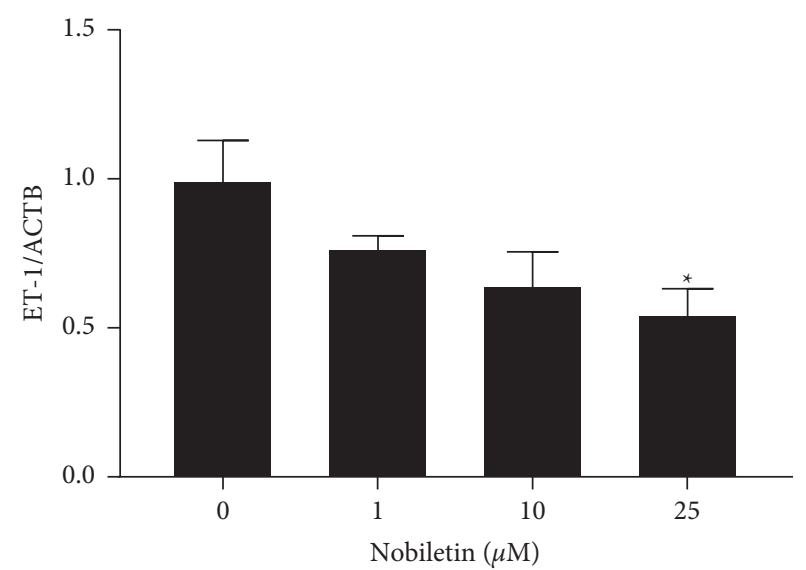

FIgURE 5: Effect of nobiletin (0-25 $\mu \mathrm{M})$ on ET-1 mRNA levels after $18 \mathrm{~h}$ incubation. Data are presented as mean \pm SD. ${ }^{*}$ indicates significant differences between the treatment group and untreated control group at $p<0.05$. 


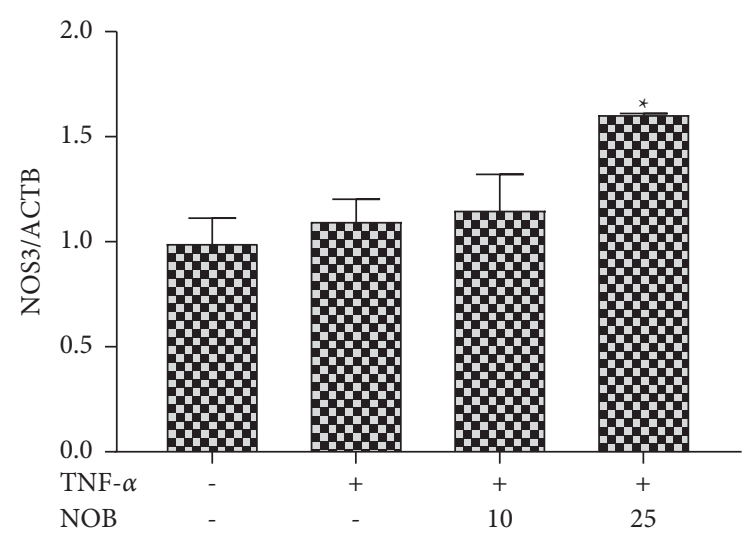

(a)

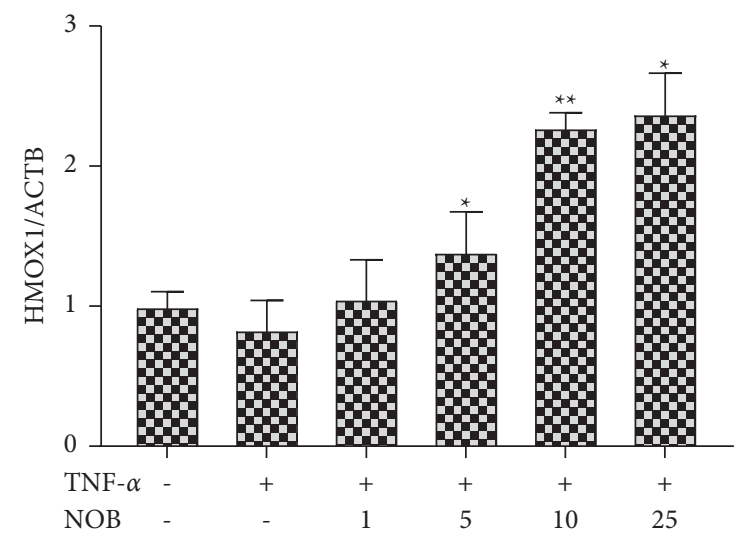

(c)

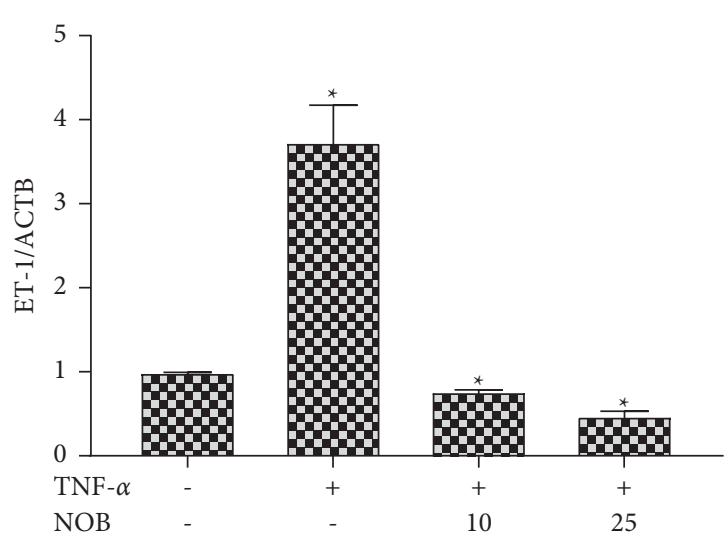

(b)

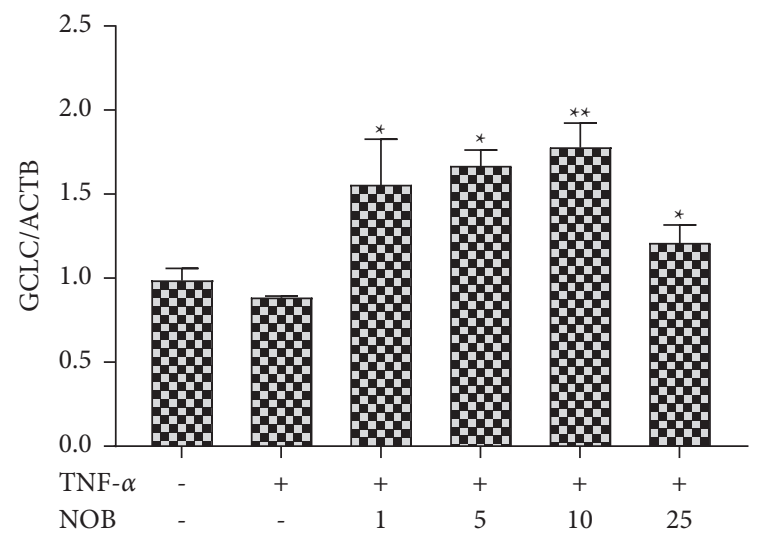

(d)

Figure 6: Effect of nobiletin (NOB) on NOS3 (a), ET-1 (b), HMOX1 (c), and GCLC (d) mRNA levels following $1 \mathrm{~h}$ pretreatment with nobiletin followed by $18 \mathrm{~h}$ incubation with TNF- $\alpha(10 \mathrm{ng} / \mathrm{mL})$. Data are presented as mean \pm SD. ${ }^{*}$ and ${ }^{* *}$ indicate significant differences between the treatment group and untreated control group at $p<0.05$ and $p<0.01$, respectively.

nobiletin may modulate endothelial cell function, since an imbalance between eNOS and ET-1 contributes to endothelial dysfunction [41].

\subsection{Effect of Nobiletin on Endothelial Cell Function under} TNF- $\alpha$-Induced Inflammatory Condition. Since nobiletin was the most promising compound in improving endothelial cell function, its effect was further investigated under TNF- $\alpha$-induced inflammatory conditions. Pretreatment with nobiletin significantly upregulated NOS3 mRNA expression by 1.6 -fold $(p<0.05)$ at $25 \mu \mathrm{M}$, following incubation of TNF- $\alpha(10 \mathrm{ng} / \mathrm{mL})$ for $18 \mathrm{~h}$ (Figure 6(a)). Meanwhile, pretreatment with nobiletin at $10-25 \mu \mathrm{M}$ significantly reduced ET-1 mRNA expression $(p<0.05)$ (Figure 6(b)). In addition, under the same investigated condition, pretreatment with nobiletin significantly increased HMOX1 (Figure 6(c)) and GCLC (Figure 6(d)) mRNA levels.

Inflammation plays a crucial role in contributing to alterations in vessel structure and function, resulting in endothelial dysfunction [42]. Elevated proinflammatory cytokines with a subsequent decrease in anti-inflammatory markers are considered as the link between inflammation and endothelial dysfunction [43]. Proinflammatory cytokine
TNF- $\alpha$ is considered to be the major inflammatory factor contributing to the development of endothelial dysfunction. Therefore, the current study aimed at investigating the effect of nobiletin on endothelial cell function under TNF- $\alpha$-induced inflammatory condition. The present study demonstrated that nobiletin was still effective in upregulating eNOS and antioxidant status-related genes as well as downregulating ET-1 under inflammatory conditions, providing new insight into how nobiletin preserves endothelial function when exposed to proinflammatory cytokines. This is of importance as inflammation contributes to the development and progression of numerous diseases such as cardiovascular disease [44], cancer [45], and neurodegenerative disease [46]. Nobiletin exerted protection against metabolic dysfunction in high-fat-fed mice and prevented obesity, hepatic steatosis, dyslipidemia, and insulin resistance [47]. Although the direct effect of nobiletin on NF- $\kappa \mathrm{B}$ pathway activation was not investigated in the present study, nobiletin previously demonstrated protective effects against LPS-induced endotoxic shock in mice, through inhibition of TNF- $\alpha$, IL-6, and high mobility group box 1 (HMGB1), through the NF- $\kappa \mathrm{B}$ signaling pathway [48]. Consistent with these findings, nobiletin was found to significantly inhibit trimethylamine oxide-induced vascular inflammation 
through inhibiting NF- $\kappa \mathrm{B}$ and MAPK/ERK-related pathways in rats [49]. Taken together, nobiletin has been shown to preserve endothelial function under inflammatory conditions by upregulation of eNOS and the Nrf-2-regulated antioxidant system as well as inhibition of ET-1.

\section{Conclusions}

In summary, different citrus flavonoids were evaluated for their effects on markers of endothelial cell function. The present data demonstrate the efficacy of nobiletin to upregulate eNOS, downregulate ET-1, and improve antioxidant status through induction of antioxidant enzymes and inhibition of cellular ROS formation, under nonstimulated and inflammatory conditions, thereby providing new evidence on eNOS protection and preservation of endothelial function. Given the potential of nobiletin, in vivo human studies, in healthy and patient cohorts, are warranted to establish in vivo bioefficacy in a safe manner, which might open new avenues of research in the development of novel natural therapeutic agents.

\section{Data Availability}

The data described in the manuscript will be made available upon request.

\section{Conflicts of Interest}

The authors declare no conflicts of interest.

\section{Acknowledgments}

Part of the work was conducted in Dr Ian Wood's laboratory. The authors are thankful for his generosity. This research was funded by the China Scholarship CouncilUniversity of Leeds Scholarship and Technological Innovation Service Capacity Building-Basic Scientific Research Expenses under grant PXM2020_014213_000017.

\section{Supplementary Materials}

The data regarding cell viability (Figure S1), effects of PMA (Figure S2), and different incubation periods (Figure S3) on NOS3 mRNA expression are demonstrated in the supplementary file. (Supplementary Materials)

\section{References}

[1] A. Cassidy, M. Bertoia, S. Chiuve, A. Flint, J. Forman, and E. B. Rimm, "Habitual intake of anthocyanins and flavanones and risk of cardiovascular disease in men," The American Journal of Clinical Nutrition, vol. 104, no. 3, pp. 587-594, 2016.

[2] D. Aune, E. Giovannucci, P. Boffetta et al., "Fruit and vegetable intake and the risk of cardiovascular disease, total cancer and all-cause mortality-a systematic review and doseresponse meta-analysis of prospective studies," International Journal of Epidemiology, vol. 46, no. 3, pp. 1029-1056, 2017.

[3] A. M. Mahmoud, R. J. Hernández Bautista, M. A. Sandhu, and O. E. Hussein, "Beneficial effects of citrus flavonoids on cardiovascular and metabolic health," Oxidative Medicine and
Cellular Longevity, vol. 2019, Article ID 5484138, 19 pages, 2019.

[4] G. R. Gandhi, A. B. S. Vasconcelos, D.-T. Wu et al., "Citrus flavonoids as promising phytochemicals targeting diabetes and related complications: a systematic review of in vitro and in vivo studies," Nutrients, vol. 12, no. 10, p. 2907, 2020.

[5] M. Wang, D. Meng, P. Zhang et al., "Antioxidant protection of nobiletin, 5-demethylnobiletin, tangeretin, and 5-demethyltangeretin from citrus peel in Saccharomyces cerevisiae," Journal of Agricultural and Food Chemistry, vol. 66, no. 12, pp. 3155-3160, 2018.

[6] W. Liao, Z. Liu, T. Zhang et al., "Enhancement of anti-inflammatory properties of nobiletin in macrophages by a nanoemulsion preparation," Journal of Agricultural and Food Chemistry, vol. 66, no. 1, pp. 91-98, 2018.

[7] R. Zhang, J. Chen, L. Mao et al., "Nobiletin triggers reactive oxygen species-mediated pyroptosis through regulating autophagy in ovarian cancer cells," Journal of Agricultural and Food Chemistry, vol. 68, no. 5, pp. 1326-1336, 2020.

[8] V. Ponzo, I. Goitre, M. Fadda et al., "Dietary flavonoid intake and cardiovascular risk: a population-based cohort study," Journal of Translational Medicine, vol. 13, no. 1, p. 218, 2015.

[9] J. R. Petrie, T. J. Guzik, and R. M. Touyz, "Diabetes, hypertension, and cardiovascular disease: clinical insights and vascular mechanisms," Canadian Journal of Cardiology, vol. 34, no. 5, pp. 575-584, 2018.

[10] H. Alhabeeb, M. H. Sohouli, A. Lari et al., "Impact of orange juice consumption on cardiovascular disease risk factors: a systematic review and meta-analysis of randomized-controlled trials," Critical Reviews in Food Science and Nutrition, pp. 1-14, 2020.

[11] L. Li, G. K. Lyall, J. A. Martinez-Blazquez et al., "Blood orange juice consumption increases flow-mediated dilation in adults with overweight and obesity: a randomized controlled trial," Journal of Nutrition, vol. 150, no. 9, pp. 2287-2294, 2020.

[12] C. Rendeiro, H. Dong, C. Saunders et al., "Flavanone-rich citrus beverages counteract the transient decline in postprandial endothelial function in humans: a randomised, controlled, double-masked, cross-over intervention study," British Journal of Nutrition, vol. 116, no. 12, pp. 1999-2010, 2016.

[13] M. Y. Schär, P. J. Curtis, S. Hazim et al., "Orange juice-derived flavanone and phenolic metabolites do not acutely affect cardiovascular risk biomarkers: a randomized, placebo-controlled, crossover trial in men at moderate risk of cardiovascular disease," The American Journal of Clinical Nutrition, vol. 101, pp. 931-938, 2015.

[14] M. G. Scioli, G. Storti, F. D’Amico et al., "Oxidative stress and new pathogenetic mechanisms in endothelial dysfunction: potential diagnostic biomarkers and therapeutic targets," Journal of Clinical Medicine, vol. 9, no. 6, p. 1995, 2020.

[15] A. Daiber, N. Xia, S. Steven et al., "New therapeutic implications of endothelial nitric oxide synthase (eNOS) function/ dysfunction in cardiovascular disease," International Journal of Molecular Sciences, vol. 20, no. 1, p. 187, 2019.

[16] P. Sharma, Y. Dong, V. K. Somers et al., "Intermittent hypoxia regulates vasoactive molecules and alters insulin-signaling in vascular endothelial cells," Scientific Reports, vol. 8, no. 1, p. 14110, 2018.

[17] R. Muniyappa, H. Chen, M. Montagnani, A. Sherman, and M. J. Quon, "Endothelial dysfunction due to selective insulin resistance in vascular endothelium: insights from mechanistic modeling," American Journal of Physiology-Endocrinology and Metabolism, vol. 319, no. 3, pp. E629-E646, 2020. 
[18] J.-G. Zou, Z.-R. Wang, Y.-Z. Huang, K.-J. Cao, and J. Wu, "Effect of red wine and wine polyphenol resveratrol on endothelial function in hypercholesterolemic rabbits," International Journal of Molecular Medicine, vol. 11, pp. 317-320, 2003.

[19] U. Förstermann and T. Münzel, "Endothelial nitric oxide synthase in vascular disease," Circulation, vol. 113, pp. 1708-1714, 2006.

[20] L. Baird and M. Yamamoto, "The molecular mechanisms regulating the KEAP1-NRF2 pathway," Molecular and Cellular Biology, vol. 40, no. 13, pp. e00099-00020, 2020.

[21] L. Li, Effects of Citrus Flavonoids on Endothelial Function and Cardiovascular Health, University of Leeds, Leeds, UK, 2017.

[22] E. Borenfreund and J. A. Puerner, "Toxicity determined in vitro by morphological alterations and neutral red absorption," Toxicology Letters, vol. 24, no. 2-3, pp. 119-124, 1985.

[23] C. Boesch-Saadatmandi, A. E. Wagner, S. Wolffram, and G. Rimbach, "Effect of quercetin on inflammatory gene expression in mice liver in vivo - role of redox factor 1, miRNA122 and miRNA-125b," Pharmacological Research, vol. 65, no. 5, pp. 523-530, 2012.

[24] J. Elíes, A. Cuíñas, V. García-Morales, F. Orallo, and M. Campos-Toimil, "Trans-resveratrol simultaneously increases cytoplasmic $\mathrm{Ca}^{2+}$ levels and nitric oxide release in human endothelial cells," Molecular Nutrition \& Food Research, vol. 55, pp. 1237-1248, 2011.

[25] H. Li, S. A. Oehrlein, T. Wallerath et al., "Activation of protein kinase $c \alpha$ and/or $\varepsilon$ enhances transcription of the human endothelial nitric oxide synthase gene," Molecular Pharmacology, vol. 53, no. 4, pp. 630-637, 1998.

[26] I. Fleming and R. Busse, "Molecular mechanisms involved in the regulation of the endothelial nitric oxide synthase," American Journal of Physiology - Regulatory, Integrative and Comparative Physiology, vol. 284, no. 1, pp. R1-R12, 2003.

[27] L. Liu, D.-m. Xu, and Y.-y. Cheng, "Distinct effects of naringenin and hesperetin on nitric oxide production from endothelial cells," Journal of Agricultural and Food Chemistry, vol. 56, no. 3, pp. 824-829, 2008.

[28] S. Hauser, F. Jung, and J. Pietzsch, "Human endothelial cell models in biomaterial research," Trends in Biotechnology, vol. 35, no. 3, pp. 265-277, 2017.

[29] Y. Deng, Y. Tu, S. Lao et al., "The role and mechanism of citrus flavonoids in cardiovascular diseases prevention and treatment," Critical Reviews in Food Science and Nutrition, vol. 2021, pp. 1-24, 2021.

[30] M. Güvenç, M. Cellat, A. Uyar et al., "Nobiletin protects from renal ischemia-reperfusion injury in rats by suppressing inflammatory cytokines and regulating iNOS-eNOS expressions," Inflammation, vol. 43, no. 1, pp. 336-346, 2020.

[31] Y. Ma, X. Ren, N. Patel et al., "Nobiletin, a citrus polymethoxyflavone, enhances the effects of bicalutamide on prostate cancer cellsviadown regulation of NF- $\kappa \mathrm{B}$, STAT3, and ERK activation," RSC Advances, vol. 10, no. 17, pp. 10254-10262, 2020.

[32] L. Martínez-Fernández, Z. Pons, M. Margalef, A. Arola-Arnal, and B. Muguerza, "Regulation of vascular endothelial genes by dietary flavonoids: structure-expression relationship studies and the role of the transcription factor KLF-2," The Journal of Nutritional Biochemistry, vol. 26, no. 3, pp. 277-284, 2015.

[33] H. Kaneda, R. Otomo, N. Sasaki, T. Omi, T. Sato, and T. Kaneda, "Endothelium-independent vasodilator effects of nobiletin in rat aorta," Journal of Pharmacological Sciences, vol. 140, no. 1, pp. 48-53, 2019.

[34] W. Yang, S. Li, L. Liao et al., "Nobiletin relaxes isolated mesenteric arteries by activating the endothelial $\mathrm{Ca}^{2+}$-eNOS pathway in rats," Journal of Vascular Research, vol. 53, no. 5-6, pp. 330-339, 2016.
[35] Z. Wang, B. Yang, X. Chen et al., "Nobiletin regulates ROS/ ADMA/DDAHII/eNOS/NO pathway and alleviates vascular endothelium injury by iron overload," Biological Trace Element Research, vol. 198, no. 1, pp. 87-97, 2020.

[36] E. Eroglu, S. S. S. Saravi, A. Sorrentino, B. Steinhorn, and T. Michel, "Discordance between eNOS phosphorylation and activation revealed by multispectral imaging and chemogenetic methods," Proceedings of the National Academy of Sciences, vol. 116, no. 40, pp. 20210-20217, 2019.

[37] D.-S. Ning, J. Ma, Y.-M. Peng et al., “Apolipoprotein A-I mimetic peptide inhibits atherosclerosis by increasing tetrahydrobiopterin via regulation of GTP-cyclohydrolase 1 and reducing uncoupled endothelial nitric oxide synthase activity," Atherosclerosis, vol. 328, pp. 83-91, 2021.

[38] K. Nohara, V. Mallampalli, T. Nemkov et al., "Nobiletin fortifies mitochondrial respiration in skeletal muscle to promote healthy aging against metabolic challenge," Nature Communications, vol. 10, no. 1, p. 3923, 2019.

[39] P. Potue, C. Wunpathe, P. Maneesai, U. Kukongviriyapan, P. Prachaney, and P. Pakdeechote, "Nobiletin alleviates vascular alterations through modulation of Nrf-2/HO-1 and MMP pathways in L-NAME induced hypertensive rats," Food \& Function, vol. 10, no. 4, pp. 1880-1892, 2019.

[40] E. E. Mulvihill, A. C. Burke, and M. W. Huff, "Citrus flavonoids as regulators of lipoprotein metabolism and atherosclerosis," Annual Review of Nutrition, vol. 36, no. 1, pp. 275-299, 2016.

[41] A. K. Ahirwar, A. Jain, A. Singh, B. Goswami, M. K. Bhatnagar, and J. Bhatacharjee, "The study of markers of endothelial dysfunction in metabolic syndrome," Hormone Molecular Biology and Clinical Investigation, vol. 24, no. 3, pp. 131-136, 2015.

[42] X. Castellon and V. Bogdanova, "Chronic inflammatory diseases and endothelial dysfunction," Aging and Disease, vol. 7, no. 1, pp. 81-89, 2016.

[43] I. K. Kwaifa, H. Bahari, Y. K. Yong, and S. M. Noor, "Endothelial dysfunction in obesity-induced inflammation: molecular mechanisms and clinical implications," Biomolecules, vol. 10, no. 2, p. 291, 2020.

[44] S. Jaiswal and P. Libby, "Clonal haematopoiesis: connecting ageing and inflammation in cardiovascular disease," Nature Reviews Cardiology, vol. 17, no. 3, pp. 137-144, 2020.

[45] R. Nagi, S. Singh, and J. Singh, "To study the effect of site and size of tympanic membrane perforation on graft uptake rates and hearing improvement in type I tympanoplasty using sliced conchal cartilage reinforced with temporalis muscle fascia and temporalis muscle fascia alone," Indian Journal of Otology, vol. 25, no. 3, pp. 121-126, 2019.

[46] M. Van Bulck, A. Sierra-Magro, J. Alarcon-Gil, A. PerezCastillo, and J. Morales-Garcia, "Novel approaches for the treatment of alzheimer's and Parkinson's disease," International Journal of Molecular Sciences, vol. 20, no. 3, p. 719, 2019.

[47] N. M. Morrow, A. C. Burke, J. P. Samsoondar et al., "The citrus flavonoid nobiletin confers protection from metabolic dysregulation in high-fat-fed mice independent of AMPK," Journal of Lipid Research, vol. 61, no. 3, pp. 387-402, 2020.

[48] W. Li, X. Wang, X. Niu et al., "Protective effects of nobiletin against endotoxic shock in mice through inhibiting TNF- $\alpha$, IL-6, and HMGB1 and regulating NF- $\kappa$ B pathway," Inflammation, vol. 39, no. 2, pp. 786-797, 2016.

[49] G. Yang, C.-C. Lin, Y. Yang et al., "Nobiletin prevents trimethylamine oxide-induced vascular inflammation via inhibition of the NF- $\kappa$ B/MAPK pathways," Journal of Agricultural and Food Chemistry, vol. 67, no. 22, pp. 61696176, 2019. 